\title{
Sobre la seguridad nacional en el discurso inaugural del Presidente Barack Obama
}

\author{
On national security in President Barack Obama's \\ inaugural speech
}

\author{
Cristian Faúndes \\ Gabriela Gallardo*
}

En este día nos reunimos porque hemos elegido la esperanza sobre el miedo; la unidad de propósitos sobre el conflicto y la discordia. Barack Obama, 20 de enero de 2009.

\section{Resumen}

En el presente artículo los autores examinan el discurso inaugural del Presidente Barack Obama intentan descifrar cuáles son los lineamientos del mandatario estadounidense respecto de la seguridad nacional, considerando que la alocución del mandatario constituye una herramienta de comunicación estratégica. Teniendo presente la problemática de las comunicaciones y su distinción con la información. Asimismo, se aplica el método de análisis de contenido por palabras, en dos etapas: cuantitativa

Cristian Faundes: Profesor civil de la Academia de Guerra del Ejército de Chile <cgfaundes@ gmail.com>.

Gabriela Gallardo: Asesora comunicacional de la Academia de Guerra del Ejército de Chile<gabigallardo@gmail.com>.

Recibido el 10 de Julio de 2009; aceptado el 19 de octubre de 2009. 
y cualitativa. Los resultados se presentan teniendo en consideración el contexto actual de seguridad nacional en Estados Unidos.

Palabras Clave: Seguridad nacional, Barack Obama, comunicación estratégica, análisis de contenido, discurso inaugural.

\section{Abstract}

In the present article, autors examine President Barack Obama's inaugural speech, with the purpose of throwing light on his ideas regarding national security, considering that the speech is a strategic communications tool and duly bearing in mind the problem of communications and its differences vis à vis information. The chosen approach is content analysis word by word, in two steps: quantitative and qualitative. Results are offered considering the present context of national security in the United States.

Key Words: National Security, Barack Obama, strategy communication, contents analysis, inagural speech. 


\section{INTRODUCCIÓN}

Uno de los ritos más connotados de la vida política en Estados Unidos es el discurso inaugural que ofrecen los mandatarios cuando juran ante la Constitución y el pueblo. El evento genera significativa expectación cuando se trata de un Presidente entrante (en vez de una reelección), más aún si asume el representante de un partido que hasta entonces fue de oposición. Desde otra perspectiva, es un momento en que los ojos del mundo observan la ceremonia con particular interés, en atención al traspaso de las riendas de la nación más poderosa del mundo.

En este contexto, la ceremonia del 2009 tuvo la particularidad de que se llevó a cabo en un momento internacional adverso para un país sumido en dos guerras (Afganistán e Irak); que encuentra una nueva rivalidad en Rusia; que tiene dificultades para controlar a Irán; que presencia las cenizas todavía humeantes de una escalada en el Medio Oriente de un conflicto asimétrico particularmente violento; mientras se forma una clara conciencia de su dependencia energética y observa la acelerada aproximación de una crisis económica mundial.

Pocos momentos son tan definitivos en la historia de una Nación, en particular cuando se entrega por primera vez el bastón de mando a un afroamericano. El hecho refleja en cierta medida el fin de más de doscientos años de discriminación racial, lo que demuestra que la gran potencia mundial está escribiendo con otro tinte su historia, dando pie a grandes cambios, tanto internos como externos.

En consecuencia, el discurso inaugural pronunciado por el Presidente Barack Obama el 20 de enero de 2009 en la ceremonia de toma de posesión del cargo en Washington D.C., es una herramienta de comunicación estratégica de gran impacto mediático. En función de ello cabe preguntarse cuáles son los planteamientos relativos a la seguridad nacional formulados por el mandatario en su mensaje inaugural.

El objetivo principal del presente trabajo, es analizar el discurso inaugural del Presidente Obama en términos de la seguridad nacional ${ }^{1}$, para lo cual, se han propuesto los siguientes objetivos específicos:

a. Identificar en términos cuantitativos cuáles son las palabras que se repiten con mayor frecuencia en el discurso.

b. Identificar qué vocablos expresados en el discurso están asociados, directamente con la Seguridad Nacional y determinar la cantidad de veces que han sido mencionados en el discurso.

c. Desarrollar un estudio cualitativo de las palabras más mencionadas en la alocución y de las palabras asociadas con la Seguridad Nacional.

1 Basado en la transcripción en vivo realizado por CNN Chile, con el apoyo de la transcripción difundida por Associated Press el 20 de enero de 2009, revisada por los autores del texto. 
d. Establecer cuál es el contenido del discurso en términos de seguridad nacional.

Para realizar el estudio se empleó el método de análisis de contenido por palabras, entendiendo que se trata de una técnica de levantamiento o recolección de información para leer e interpretar el contenido de toda clase de documentos escritos o de otra índole.

En primera instancia se identifican los seis vocablos que fueron pronunciados con mayor frecuencia en el discurso, para luego buscar en forma específica la presencia de términos relacionados con la seguridad nacional.

En la segunda parte del estudio se realiza un análisis cualitativo de las seis palabras más mencionadas en el discurso, así como de aquellas asociadas con la seguridad que han sido identificadas por los autores. Lo cualitativo corresponde a la interpretación de los investigadores, teniendo presente la mención de las palabras en su contexto, así como su relación con la situación actual que vive Estados Unidos.

Los resultados principales del análisis indican que la alocución del mandatario estadounidense aborda la seguridad nacional en un sentido amplio, orientado más al desarrollo que a la defensa. Esto se expresa en la única mención explícita del vocablo "security» en que manifestó que en su opinión la seguridad no consiste solamente en el poderío militar sino que incluye otros factores fundamentales, como la justicia de la causa estadounidense, la fuerza del ejemplo y las cualidades atenuantes de la humildad y la moderación. En este contexto, propone planteamientos más conceptuales que concretos, formula una amplia invitación al pueblo a emprender una marcha unida para enfrentar las dificultades del presente, en contraste con las necesidades de gestionar las crisis.

Con todo, no se trata de juzgar un discurso presidencial, sino de interpretar lo que el mandatario declara atendiendo a las utilidades comunicacionales que brinda el momento histórico y las expectativas generadas.

\section{El DisCURSO COMO}

HERRAMIENTA COMUNICACIONAL

Aunque el discurso como herramienta comunicacional constituye un tema de investigación en sí, para efectos del presente estudio se puede acotar que en esencia la palabra es un instrumento de comunicación, de modo que el lenguaje permite expresar pensamientos, información y afectos. En este contexto, el discurso es una forma organizada de expresión dirigido a un grupo de auditores. La finalidad del emisor del mensaje puede ser diversa, en especial cuando la alocución obedece a un objetivo político.

En consecuencia, en el presente trabajo se realiza un ejercicio metódico con la intención de identificar la información relativa a la seguridad nacional que subyace el efecto comunicacional del texto definido. Para ello, es fundamental 
considerar que el discurso pronunciado por el Presidente Barack Obama en la ceremonia de toma de posesión del cargo se constituye en herramienta comunicacional que tiene un propósito político al aprovechar la oportunidad que le brindan las expectativas de la coyuntura y el momento político. Mayor significación tiene la difusión masiva del evento para una cultura uno de cuyos pilares es la confianza. Entonces, el discurso presidencial inaugural no trata únicamente de un deber cívico sino que consiste en la colocación de la primera piedra fundacional de una administración que se da a conocer. Por tanto, en la ceremonia el mandatario transmite su carácter, así como el de la administración entrante. Con todo, a grandes rasgos el discurso inaugural es un ejercicio de comunicación estratégica.

En términos generales, propiamente se entiende como comunicación estratégica (Tironi y Cavallo, 33; 2006) «una herramienta disponible para organizaciones de cualquier naturaleza: empresas, fundaciones, entidades de gobierno, instituciones religiosas, grupos y marcas (...) la tarea es proyectar la identidad de las organizaciones en una imagen que suscite confianza en su entorno relevante y adhesión en su público objetivo».

Tironi y Cavallo (2006) explican que la comunicación estratégica responde a la necesidad actual de gobiernos y en general entidades privadas y públicas que requieren herramientas eficaces para llegar al público que desean cautivar, mediante una entrega informativa que se puede emitir a través de diversos canales comunicacionales.

Según comentan Tironi et. al. (29; 2006):

Los gobiernos contemporáneos establecen buena parte de su agenda y sus actuaciones en función de los medios de comunicación, para influir sobre los actores institucionales y sociales relevantes así como sobre la ciudadanía.

En el siglo 21, cuando los gobiernos ya no tienen control sobre la producción ni sobre los servicios sociales (como efecto de las privatizaciones), ni sobre sus políticas monetarias, exterior y de defensa (como efecto de la globalización), su poder se expresa básicamente en su capacidad para emitir señales a través de los medios de comunicación...

Ahora bien, es fundamental expresar que la aproximación al estudio se ejecuta considerando la distinción entre comunicación e información.

Comunicación es la acción y efecto de comunicar o comunicarse, implica la transmisión de mensajes y la recepción de los mismos en el seno del grupo, considerada en sus relaciones con la estructura de este grupo. Es exitosa en la medida en que la transmisión de señales se realice mediante un código común al emisor y el receptor. Pero el término tiene una incidencia más profunda. Según afirma Pellegrini (24; 1993): «comunicación es una necesidad del hombre, que tiene directa relación con sus características esenciales de sociabilidad. A través de la comunicación no solo se integra el hombre a la sociedad, sino que esta misma, se hace 
recién posible». Es lo que posibilita la interacción en un grupo en cuanto permite compartir lo propio y lo ajeno. Explica Pellegrini $(25 ; 1993)$ «es la comunicación, entonces, de donde surgen nociones como bien común, nación y patria, por ejemplo».

En la comunicación se pueden distinguir distintas vertientes o planos de acción entre las que se reconocen las comunicaciones de carácter afectivo, las generadoras de opinión y las propiamente informativas. Según explica Pellegrini $(28 ; 1993)$ «generalmente las tres se producen en forma integrada en cada persona, en cada medio e, incluso, en ciertos mensajes».

Podemos decir entonces que la información se encuentra al interior de la comunicación como elemento particular que permite que el receptor conozca hechos y comprenda el entorno que le rodea, dándole la posibilidad de aumentar sus conocimientos.

Por lo que respecta a la connotación del mensaje analizado del mandatario de la nación más poderosa del mundo, difundido en vivo por cadenas de televisión a todo el planeta, es pertinente recoger dos conceptos que hace presente María Dolores Montero (1998) desde las Ciencias de las Comunicaciones. Por una parte, la "aguja hipodérmica», que se basa en el supuesto de que los mensajes de los medios de comunicación afectan directamente a cada individuo y pueden provocar cambios de opinión. Por otro lado, existe el «flujo de comunicación en dos etapas», que describe cómo la información pasa por los líde- res de opinión y desde ellos a los grupos que conforman la sociedad.

Teniendo presente el alto nivel de accionar que tienen comunicación e información dentro de la sociedad, debe procurarse destacar que el análisis de contenido realizado se basa, justamente, en la información extractada de un proceso puntual de comunicación.

Por ello, y como bien define Ruiz (192: 2003), el análisis de contenido es «una técnica para leer e interpretar el contenido de toda clase de documentos y, más concretamente (aunque no exclusivamente) de los documentos escritos». El autor explica que se basa en la lectura como instrumento para reunir información; lectura que debe realizarse de modo científico, es decir, de manera sistemática, objetiva, replicable, válida.

La finalidad de la técnica de análisis de contenido, explica Klaus Krippendorff (28: 1997) «consiste en proporcionar conocimientos, nuevas intelecciones, una representación de los «hechos» y una guía práctica para la acción. Es una herramienta».

Utilizamos el análisis de contenido, con la finalidad de interpretar el discurso (Ruiz, 193: 2003) «Conforme a los postulados del paradigma constructivista, entiende que es el propio investigador el que crea, a través de una serie de prácticas interpretadoras, los materiales y la analiza, posteriormente, su evidencia. Toda redacción de un texto y toda lectura posterior del mismo (entendida como recogida de información), son, al mismo tiempo, una construcción social y política». 
Asimismo, al captar el contenido de un escrito se extraen inferencias del mismo. Ruiz (195: 2003) explica que «La inferencia es un elemento central del análisis del contenido. El análisis de contenido se distingue del análisis documental en que este último se limita estrictamente al contenido del texto mismo, mientras que el primero elabora, a partir del texto, inferencias sobre el contexto del mismo».

Es fundamental tener presente que todo texto puede tener una doble lectura. Ruiz (195: 2003) considera que están: «la directa del sentido manifiesto, al pie de la letra y la soterrada del sentido latente, entre sacado del otro». Teniendo presente, como bien dice este autor, que (Ruiz, 196-197: 2003) «los mensajes y, en general, la comunicación simbólica, expresan (contienen, dicen, manifiestan) más cosas que el propio autor del texto pretende decir o de las que él mismo es consciente».

Recogiendo esta última afirmación, los investigadores del presente estudio reconocen que un mensaje es capaz de transmitir una multiplicidad de contenidos incluso a un receptor único. En este sentido, se considera a Krippendorff (30: 1997) cuando señala que «en estas circunstancias, la pretensión de haber analizado el contenido de la comunicación trasluce una posición insostenible».

$\mathrm{El}$ análisis que se desarrolla en este caso particular incorpora dos etapas fundamentales a destacar: análisis cuantitativo y análisis cualitativo.

Para efectuar el análisis cuantitativo se generan datos a partir de la revisión general del texto y de la búsqueda de las palabras más mencionadas. Así se obtiene como resultado un número que refleja la frecuencia con la que cada palabra ha sido nombrada, es decir, un dato.

Sin embargo, es primordial destacar, que la recolección de datos en sí (o su producción) no es suficiente para obtener conclusiones de un estudio determinado. Según explican Rodríguez, Gil y García (200: 1999) «los datos no son más que un material bruto a partir del cual el investigador debe realizar las operaciones oportunas que le llevan a estructurar el conjunto de información en un todo coherente y significativo».

En este sentido, se justifica efectuar en el presente estudio un análisis cualitativo, considerando que se pretende descifrar el significado real de estos datos, para obtener una información idónea y pertinente, que nos posibilite interpretar la visión del mandatario sobre la seguridad.

En la etapa cualitativa, en la cual se analizan datos obtenidos en la etapa anterior, se desarrolla un proceso que se define como (Rodríguez y otros 200; 1999) «un conjunto de manipulaciones, transformaciones, operaciones, reflexiones, comprobaciones que realizamos sobre los datos con el fin de extraer significado relevante en relación a un problema de investigación». Rodríguez y otros $(200 ; 1999)$ comentan que «En efecto analizar datos supondrá examinar sistemáticamente un conjunto de elementos informativos para delimitar partes y descubrir las relaciones entre las mismas y las relaciones con el todo». 
En este punto corresponde anotar que existen vínculos entre la comunicación y la seguridad nacional que se expresan en el lenguaje o, dicho de otra manera, se manifiestan en la generación de un metalenguaje. Esto puede observarse a propósito de la denominada «lucha contra el terrorismo» después de que se descubre la existencia de abusos cometidos contra prisioneros sospechosos de ejecutar acciones de terrorismo.

Berkowitz (21: 2008) comenta, "The Abu Ghraib photos changed the entire momentum of the war in Iraq. Public support for the war plummeted among America and its allies. Reactions in the Muslim world were even worse».

A juicio de Priest (23 de enero, 2009), los congresistas demócratas estadounidenses que ejercieron su cargo durante el gobierno de Bush, «replaced the «war on terror» phraseology with language indicating vigilance and persistence, but not unending combat and military-only options».

En su discurso inaugural, el Presidente Obama (Obama a, 20 de enero, 2009) utiliza distintos términos para referirse a la lucha: "As for our common defense, we reject as false the choice between our safety and our ideals».

A propósito de la misma alocución, Cohen (22 de enero, 2009) comenta la ausencia de la frase "guerra global contra el terror» explicando que «There is no point in flattering America's adversaries by inflating their vile methods into an ideology and then declaring an unwinnable war against it».
En términos generales, se señala que los demócratas, y en especial la nueva administración de gobierno de los Estados Unidos, han adoptado un nuevo léxico para referirse al conflicto con los terroristas.

Con todo, el contexto es esencial como elemento que nutre las expectativas y alimenta las perspectivas para la formulación del análisis cualitativo. Básicamente fundamenta la preparación del estudio en atención a la situación de la seguridad nacional en los Estados Unidos.

\section{LAS CUATRO CRISIS}

A nuestro juicio, lo principal del contexto de seguridad en los Estados Unidos se expresa en cuatro crisis simultáneas:

1. La primera de ellas se relaciona con las dos guerras en que está involucrado el país, en Afganistán e Irak.

2. En segundo término, se destaca la crisis económica provocada por los créditos inmobiliarios en Estados Unidos y su propagación al sistema financiero mundial, que se extendió a los diversos campos de la economía y a motros sectores en lo que muchos especialistas consideran la peor crisis desde la depresión de 1930. Al respecto, un editorial del Washington Post (20 de enero, 2009) comenta "an economy in tatters poses an immediate challenge on a scale not faced since Franklin 
Roosevelt took office in the midst of the Great Depression».

3. En tercer lugar, cabe mencionar la crisis de aliados como factor importante de tener presente, considerando el desarrollo de la política exterior de Estados Unidos en el gobierno anterior, y la consiguiente pérdida de apoyo en el sistema internacional.

4. Finalmente, cabe mencionar la crisis de confianza al interior de Estados Unidos, tal vez la más peligrosa y menos nombrada, cuya manifestación más evidente es el desmembramiento del tejido social y la ruptura de la relación entre el gobierno y el pueblo. Esto último se refleja en el bajo índice de apoyo al mandatario saliente que según Gallup (14 de enero, 2009) alcanzó un mínimo de $25 \%$ de apoyo en noviembre de 2008 , justo antes de las elecciones y un $34 \%$ de aprobación y un $61 \%$ de rechazo en la encuesta de USA Today/Gallup realizada los días 9 al 11 de enero de 2009.

Nada de lo anterior opera en forma aislada, sino que se potencia en forma negativa generando presiones de distinta índole, lo que para algunos académicos y especialistas implica el declive del país como potencia unipolar. Al respecto, cabe tener en cuenta un comentario de Cohen (22 de enero, 2009), «But engaged in two wars, its Treasury bare, the United States has a pressing strategic interest in furthering peace where it can».

Desde una perspectiva más específica, el analista George Friedman observa que la guerra en Afganistán constituye en sí un serio problema, en cuanto presiona a Estados Unidos a desarrollar condiciones para generar un corredor alternativo a Pakistán para abastecer a las tropas. Sostiene que esta situación se soluciona sólo con el apoyo de Rusia, complejizando el asunto. A su juicio, entre la guerra en Afganistán y la crisis económica (Friedman, 19 de enero, 2009) "we will find out what kind of president Obama is».

Desde una mirada a la situación interna de Estados Unidos, este discurso tiene una connotación social que es sumamente importante destacar, por cuanto se trata del primer presidente de origen afroamericano en asumir el poder. Cabe destacar que esto indica un progreso sustantivo en términos de la historia de la segregación racial y de los derechos civiles en el país.

El propio Obama se refirió al tema la semana antes de asumir el cargo como mandatario (Washington Post, 20 de enero, 2009): «There is an entire generation that will grow up taking for granted that the highest office in the land is filled by an African American... . It changes how black children look at themselves. It also changes how white children look at black children. And I wouldn't underestimate the force of that».

Además, es importante tener presente que, como ya se señaló, el discurso se pronuncio en una delicada situación de crisis. Al respecto comenta Gellman (20 de enero, 2009), «Not since Reagan have we had as capable a persuader as Obama, and not since FDR has a president 
come in with quite the configuration of foreign and domestic crises that open up such a possibility for the reconstruction of the executive». A lo anterior se suma su popularidad, reflejada en el alto nivel de aprobación que consigue al inicio de su mandato: $68 \%$. La cifra (Jones, 26 de enero, 2009) lo posiciona en el segundo lugar de preferencias (detrás del Presidente Kennedy quien el año 1961 obtuvo un $72 \%$ ) entre los nueve mandatarios que han asumido por primera vez el cargo desde 1953, año en que Gallup comenzó a elaborar la encuesta.

En el contexto mundial, el discurso es relevante por las expectativas que tienen los demás países por conocer cómo el nuevo presidente de Estados Unidos va a enfrentar los desafíos que se le presentan en el concierto internacional y la relación que existirá entre la primera potencia mundial y los demás países del orbe.

Aquí no solo se trata de las crisis en que Estados Unidos está directamente envuelto, sino también de los desafíos globales, tales como el conflicto árabeisraelí (y sus violentas expresiones en Gaza a principios del 2009); la proliferación nuclear en Irán y Corea del Norte; la tensión entre India y Pakistán; las situaciones de conflicto interno en Darfur, Myanmar, la República Democrática del Congo y Zimbabwue; el calentamiento global; y el terrorismo internacional.

En suma, las cuatro crisis identificadas configuran un contexto que genera amplias expectativas dada la incertidumbre asociada a esos fenómenos. Por tanto, interpretar del texto fundacional de la administración del Presidente Barack Obama se hace una necesidad que se procura en cierto modo satisfacer con el estudio que figura a continuación.

\section{Desarrollo}

El estudio permite determinar que en términos cuantitativos el discurso tiene un fuerte énfasis en la comunidad de ciudadanos estadounidenses, con un $6,34 \%$ de alusiones directas, a diferencia de los términos asociados con la seguridad, que en conjunto representan un $1,17 \%$ del total de la disertación.

Los resultados cualitativos permiten observar la relevancia que el mandatario otorga a los valores patrios, que dan fundamento al llamado a la unidad estadounidense, y que va dirigido a un conglomerado que a su juicio puede sobrepasar la adversidad como lo ha hecho antes. Finalmente, en cuanto a la seguridad, propone efectuar un giro desenfundando las armas de la diplomacia para ejercer un liderazgo mundial desde la responsabilidad.

\subsection{Resultados cuantitativos}

El Presidente Barack Obama inauguró su mandato el 20 de enero de 2009 en Washington D.C. pronunciando un discurso de $2.393^{2}$ palabras en poco menos de

2 La transcripción del discurso difundido por Associated Press tiene tres palabras menos que las emitidas originalmente por el mandatario. 
20 minutos. Los tres vocablos más mencionados son Our (67), We (62) y Us (23).

Para efectos del presente estudio, es interesante constatar que "Our» representa un $2,79 \%$ del total de expresiones contenidas en el mensaje presidencial, «We» el 2,59\% y «Us» el 0,96\% Como se puede observar, los tres términos, se refieren a una misma entidad o sujeto colectivo, comunidad de individuos que en la expresión "Our» contiene una calidad posesiva. En suma, el Presidente Obama, en un $6,34 \%$ del discurso habla directamente a una entidad colectiva de ciudadanos, como puede verse en el Gráfico $\mathrm{N}^{\circ} 1$.

\section{Gráfico No i. Resultados cuantitativos}

Palabras asociadas con una comunidad de individuos como parte del total de palabras del discurso inaugural del Presidente Barack Obama pronunciado el 20 de enero de 2009.

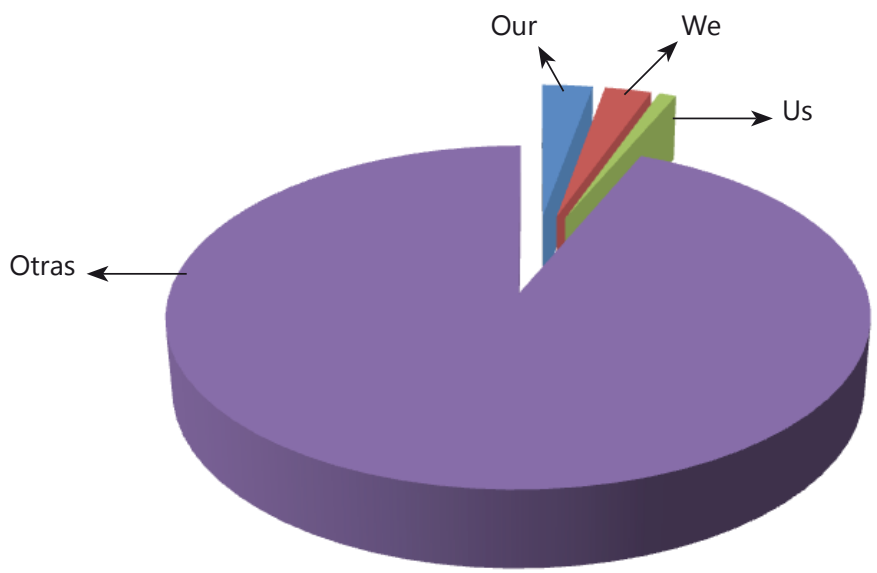

Fuente: Elaborado por los autores a partir de los resultados del estudio.

Por lo que respecta a la colectividad de ciudadanos, la palabra "Can» tiene a su vez trece menciones, de diversa significación. En una mirada en detalle, se observa que en ocho ocasiones la expresión se utiliza en términos positivos relacionados con una capacidad o posibilidad de acción de los distintos actores del país. En otras cinco oportunidades, se emplea en términos negativos o relacionados con otros actores del extranjero ${ }^{3}$. Asociado a esta expresión, se encuentra «capacity», para expresar

3 «...the market can spin out of control» «Our Founding Fathers, faced with perils that we can scarcely imagine». "To those leaders around the globe who seek to sow conflict, or blame their society's ills on the West -know that your people will judge you on what you can build, not what you destroy». «...we can no longer afford indifference to the suffering outside our borders». «...nor can we consume the world's resources without regard to effect». 
que las capacidades del país no han menguado a pesar de la crisis.

De igual forma, el mandatario hace doce referencias directas a la nación estadounidense mediante el vocablo «Nation» y utiliza el término «naciones», en relación con la búsqueda de una mayor cooperación y entendimiento entre las naciones; respecto de las naciones pobres; $y$ las relaciones de
Estados Unidos con las naciones que tienen relativa abundancia.

La palabra «America» se menciona diez veces y en todas ellas el mandatario se refiere a Estados Unidos (no al continente como podría esperar la mayoría de los habitantes del hemisferio). Asociado a esta expresión se encuentra "American» que tiene otras cinco menciones.

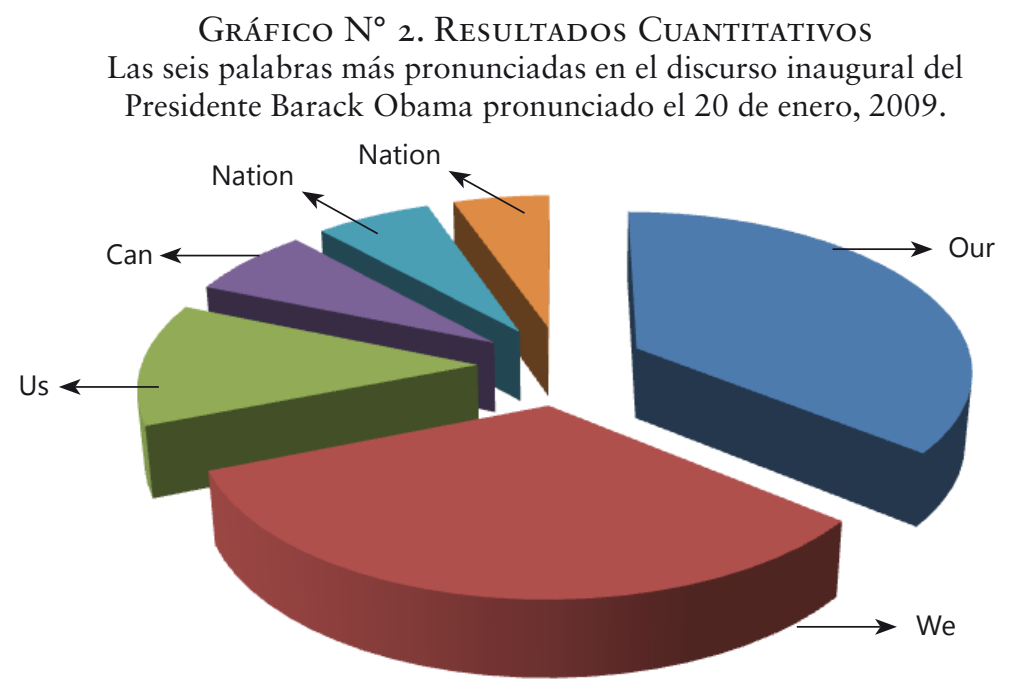

Fuente: elaborado por los autores con los resultados del estudio.

En tanto, se encontraron otras 28 menciones de palabras vinculadas a la seguridad nacional, que representan el $1,17 \%$ del total del discurso, según se detalla a continuación:

Power: 4. En dos ocasiones, para destacar la relación entre el poder de la nación estadounidense y la responsabilidad asociada con su utilización. En una oportunidad, para destacar que quienes utilizan el poder de la corrupción y del engaño para silenciar a la disidencia, se encuentran en el lado equivocado de la historia. Además, para resaltar que a pesar de la crisis, Estados Unidos tiene más poder que cualquier otra Nación para generar riqueza y ampliar la libertad. Asociado a la locución se encuentra Powerful, cuya única mención figura en una frase en que el mandatario destaca que Estados 
Unidos aún es la nación más poderosa del planeta.

Peace: 4. En el segundo párrafo, a modo introductorio, se menciona esta palabra, para destacar que el juramento de los 44 presidentes de Estados Unidos se ha efectuado en tiempos de paz y en momentos de dificultades. De igual forma, usa este vocablo al señalar que Estados Unidos busca un futuro de paz y dignidad, como asimismo, en relación con la construcción de la paz en Afganistán y a propósito de que los Estados Unidos deben desempeñar un papel en la creación de una nueva era de paz.

Freedom: 3 Liberty: 2. En el presente trabajo ambos términos se consideran como sinónimos. Freedom se emplea junto con prosperity para señalar la dirección del camino que siguen los estadounidenses (un duro camino a la prosperidad y la libertad). Además, la palabra se relaciona con el poder, como se dijo, para ampliar la libertad y como un regalo que se entregará a los nietos, que en el futuro recordarán que ante la puesta a prueba de la generación actual, los estadounidenses rehusaron interrumpir su viaje a la libertad. En tanto, la palabra liberty se menciona cuando se rinde homenaje a los héroes que, juicio del mandatario, además de guardianes de la libertad, encarnan el espíritu de servicio y, refiriéndose al significado de la libertad y el credo estadounidense, que la nación se sustenta en la fe y la determinación de su pueblo.

Friend: 3. La palabra se menciona a propósito de la declaración de que los
Estados Unidos son amigos de cada nación o individuo que busque un futuro de paz y dignidad. Sin embargo en la misma oración, Estados Unidos se sitúa en posición de liderazgo y llama a los amigos del país a trabajar en conjunto para enfrentar peligros globales como la amenaza nuclear y el cambio climático. Finalmente para mencionar la amistad individual en función de la fe y la determinación de la ciudadanía en que se sustentan la nación americana.

Threat: 3. En el cuarto párrafo, utiliza esta palabra a propósito del modo en que Estados Unidos emplea la energía que fortalece a los adversarios del país y amenaza al planeta. También la usa en relación con los principios que guían a la nación para enfrentar las nuevas amenazas, explícitamente las dos guerras en Irak y Afganistán, la proliferación nuclear, el calentamiento global y el terrorismo y en último término, la amenaza nuclear.

War: 2. En el cuarto párrafo, cuando afirma que la nación se encuentra en guerra contra una red de violencia y odio de largo alcance, como asimismo, cuando menciona la guerra civil estadounidense como una de las amargas experiencias (junto con la segregación) de la que Estados Unidos ha surgido fortalecido. 
Gráfico $\mathrm{N}^{\circ} 3$. Resultados cuantitativos

Palabras asociadas con la seguridad como parte del total de palabras del discurso inaugural del Presidente Barack Obama pronunciado el 20 de enero, 2009.

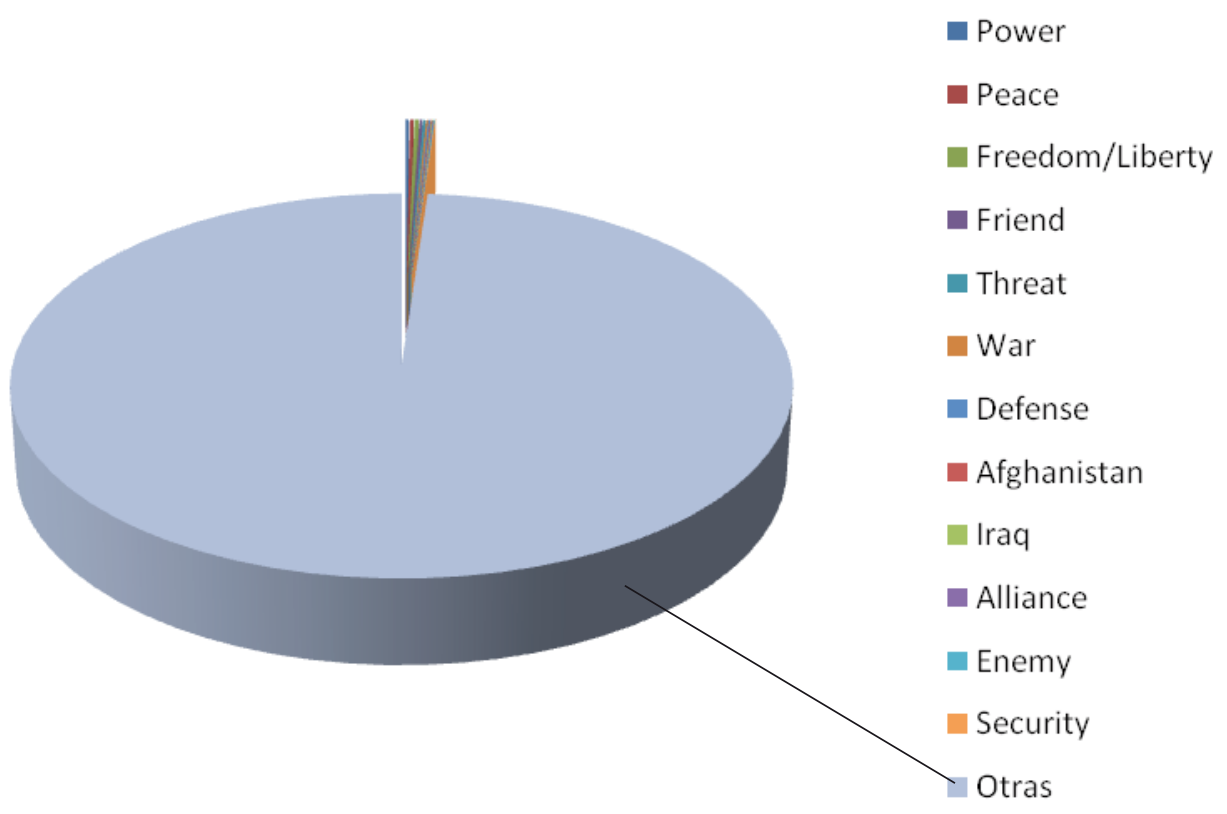

Fuente: elaborado por los autores a partir de los resultados del estudio.

Defense: 2. Respecto de la defensa común, el Presidente Obama rechaza la disyuntiva entre seguridad e ideales de la Nación, tales como el imperio de la ley y los derechos humanos. En la segunda ocasión menciona la palabra al declarar que Estados Unidos no vacilará en defender el modo de vida estadounidense.

Afghanistan: 1 . Se pronuncia a propósito del interés por construir la paz en ese país.

Iraq: 1 . Anunciando un retiro responsable para entregar el país a su gente.

Alliance: 1 . La única ves que menciona este vocablo es a propósito de la alianza que hicieron generaciones pasa- das para enfrentar los grandes desafíos del comunismo y el fascismo y destacar que esa flagelos han sido superados con algo más que tanques y misiles, con alianzas y convicciones fuertes.

Enemy: 1. La palabra se menciona al recordar un hito en la lucha independentista de Estados Unidos cuando las malas condiciones de los revolucionarios y el avance del enemigo ponían en duda las posibilidades de la revolución. No se menciona el vocablo en plural.

Security: 1 . Este vocablo está sujeto a la relación con el poder, para declarar que la seguridad del país emana de otros factores además del poder militar. En sus palabras (Obama a, 20 de enero, 
2009): «from the justness of our cause, the force of our example, the tempering qualities of humility and restraint».

\subsection{Resultados cualitativos}

En cuanto al análisis cualitativo, las seis palabras más mencionadas en el discurso inaugural se han relacionado en tres grupos: a.-Our, We, Us. b.- Nation, America. c.- Can (capacidad para hacer). Un cuarto grupo de análisis está compuesto por otros vocablos expresados en el discurso directamente vinculados con la seguridad nacional: d.- Afghanistan, Alliance, Defense, Enemy, Freedom, Friend, Iraq, Peace, Power, Security, Threat, War.

El primer grupo, incorpora las palabras que indican el énfasis en la mirada a lo común, la dirección de la palabra a una entidad única, la comunidad de ciudadanos estadounidenses.

El segundo, es el objeto del discurso, «America», la «Nación» estadounidense, por tanto su relevancia es inobjetable.

El tercer grupo se relaciona con el vocablo «can» y tiene que ver con el poder del ciudadano para hacer un cambio necesario según lo indica una de las palabras lema de la campaña del mandatario: «Change». Justamente es la capacidad, como veremos junto con la esperanza, en la que se sustenta otro de los íconos de la elección: «Yes, we can».

Finalmente, el último, se estructura en función del objeto principal del presente estudio, que es esbozar una lectu- ra del discurso inaugural del Presidente Obama desde la óptica de la seguridad, teniendo en cuenta particularmente, que en el discurso analizado el término solo se utiliza una vez.

\subsubsection{Our, We, Us}

Justamente las tres palabras más mencionadas en el discurso aluden a la unidad nacional, reforzando la intencionalidad del mensaje. No se puede perder de vista que el mandatario destina el $6,34 \%$ de su alocución a mencionar alguno de estos tres vocablos.

La piedra angular es la gente, en específicamente el pueblo norteamericano, que debe enfrentar los desafíos del presente. En torno a la nación -como ente forjado por los ciudadanos- giran los elementos, las tareas, los desafíos inminentes que tiene la nueva administración, considerando el escenario en el cual se está desarrollando el nuevo gobierno.

Por medio del discurso se busca generar una comunidad de intenciones y objetivos. Al respecto surge la responsabilidad como cargo u obligación moral de los ciudadanos, no solo para las futuras generaciones sino para honrar la memoria de quienes han sacrificado su vida para que Estados Unidos sea la nación más próspera y poderosa del planeta. De este modo, es la unidad de America-como dicen los estadounidenses- la que da forma al país, Estados Unidos. Básicamente, el nombre del país conceptualiza la unidad. 
La situación se determina por las cuatro crisis que afectan al país, pero sensiblemente la crisis de confianza al interior de Estados Unidos, la más peligrosa y menos nombrada es la que a juicio del Presidente Obama amerita recordar cual es el silencioso motor del progreso (Obama a, 20 de enero, 2009): "Our challenges may be new. The instruments with which we meet them may be new. But those values upon which our success depends -honesty and hard work, courage and fair play, tolerance and curiosity, loyalty and patriotismthese things are old. These things are true. They have been the quiet force of progress throughout our history».

En este contexto es interesante recoger la percepción que Gail Collins (21 de enero, 2009) presenta en The New York Times comparando la experiencia de Woodstock con la ceremonia, afirmando que en ambas: "there was a wonderful feeling of community». Agrega que la diferencia se encuentra en la reacción a nivel nacional, explica que mientras en Woodstock la unidad se limitó a los asistentes al evento (Collins, 21 de enero, 2009) "the inauguration left the whole country glued together emotionally, one big American ball of hope».

En contraste, Cohen (22 de enero, 2009) declara: «I sat 30 feet away and felt stirred but not transported. Perhaps that was the point. There's too much work to do for high rhetorical flourish».

\subsubsection{Nation, America, American}

El término nación, entonces, surge en función de una responsabilidad compartida que no se limita al escenario interno sino que se extiende al orbe como la nación más próspera y más poderosa de la Tierra.

El mandatario considera que el norteamericano, como ciudadano, debe aportar su esfuerzo al bien del país y de la humanidad (Obama a, 20 de enero, 2009): "What is required of us now is a new era of responsibility - a recognition, on the part of every American, that we have duties to ourselves, our nation, and the world, duties that we do not grudgingly accept but rather seize gladly, firm in the knowledge that there is nothing so satisfying to the spirit, so defining of our character, than giving our all to a difficult task».

El mandatario hace un llamado a enfrentar los nuevos desafíos que se presentan como consecuencia de una crisis que ha hipotecado el futuro de las nuevas generaciones. Ante ello, Obama sostiene que el país no ha perdido sus capacidades, por tanto, a su juicio se trata de una crisis que se debe afrontar como nación.

La nación la integran todos. En este contexto es clave que las relaciones entre el gobierno y el pueblo se expresen en un marco de confianza, particularmente en un sistema democrático. Al respecto, el Presidente Obama le dedica un párrafo completo de su discurso inaugural, probablemente debido a la incidencia en la crisis de confianza al interior de Estados Unidos. Afirma (Oba- 
ma a, 20 de enero, 2009): "And those of us who manage the public's dollars will be held to account -to spend wisely, reform bad habits, and do our business in the light of day- because only then can we restore the vital trust between a people and their government».

Pero más allá del gobierno, consigna que la nación es de todos (Obama, 20 de enero, 2009): "America has carried on not simply because of the skill or vision of those in high office, but because We the People have remained faithful to the ideals of our forbearers, and true to our founding documents». Estos documentos fundacionales institucionalizan la formación de la nación que ha llegado donde está gracias a su gente.

Asimismo, la heterogeneidad del país queda expresada en términos positivos como un tejido social que incluye cristianos, musulmanes, judíos e hindúes así como no creyentes, cuando declara que (Obama a, 20 de enero, 2009):

We are a nation of Christians and Muslims, Jews and Hindus - and non-believers. We are shaped by every language and culture, drawn from every end of this Earth; and because we have tasted the bitter swill of civil war and segregation, and emerged from that dark chapter stronger and more united, we cannot help but believe that the old hatreds shall someday pass; that the lines of tribe shall soon dissolve; that as the world grows smaller, our common humanity shall reveal itself; and that America must play its role in ushering in a new era of peace.
Según informa Kristof (21 de enero, 2009), el Presidente Obama es el primero en emplear la palabra «musulmán» en un discurso inaugural.

De este modo, a diferencia del gobierno anterior marcado por una ideología restringida basada en el cristianismo, se presenta un esquema de unidad. Cohen (22 de enero, 2009) comenta al respecto: «a far less ideological United States will emerge under Obama, one that will speak less of a global mission to spread liberty, democracy and free markets. Call it the new American humility».

Finalmente, para el mandatario estadounidense, se trata de superar las propias barreras internas de una nación que progresa hacia la inclusividad (Obama a, 20 de enero, 2009): "This is the meaning of our liberty and our creed - why men and women and children of every race and every faith can join in celebration across this magnificent mall, and why a man whose father less than sixty years ago might not have been served at a local restaurant can now stand before you to take a most sacred oath».

Básicamente se trata de colocar en la agenda social una unidad de propósito que se encuentra en el establecimiento de la confianza entre el gobierno y el pueblo ${ }^{4}$, el reunir la diversidad de culturas y creencias en torno a un bien común, y recoger la herencia histórica de quienes forjaron un país, a juicio del

4 Asunto clave como sustento de un sistema democrático. 
orador, enfrentando las más difíciles circunstancias.

\subsubsection{Can}

Según el mandatario, quienes cuestionan la capacidad de Estados Unidos, lo hacen porque (Obama a, 20 de enero, 2009): «For they have forgotten what this country has already done; what free men and women can achieve when imagination is joined to common purpose, and necessity to courage».

El mandatario se refiere a la capacidad de cada uno de los norteamericanos, que ha sido afectada no por las crisis actuales sino por el inmovilismo, el proteger estrechas decisiones y aplazar decisiones desagradables. La esperanza ha sido menoscabada y se ha infundido el miedo, el conflicto y la discordia en la agenda social, trabando la determinación para enfrentar nuevos retos.

Por tanto, esta capacidad debe estar resguardada ante todo por una esperanza social. "Hope», más que lema de campaña se convierte entonces en un concepto principal que ilumina el desarrollo de la agenda social con miras al progreso y a levantar el país. Finalmente, es la respuesta para resolver este problema denominado crisis. En este contexto uno de los párrafos más significativos es el siguiente (Obama a, 20 de enero, 2009):

We remain the most prosperous, powerful nation on Earth. Our workers are no less productive than when this crisis began. Our minds are no less inventive, our goods and services no less needed than they were last week or last month or last year. Our capacity remains undiminished. But our time of standing pat, of protecting narrow interests and putting off unpleasant decisions - that time has surely passed. Starting today, we must pick ourselves up, dust ourselves off, and begin again the work of remaking America.

Finalmente el Presidente Obama declara que el lugar de Estado Unidos. en el mundo actual no es resultado del azar sino fruto del esfuerzo de los ciudadanos que se han sacrificado por el país. La capacidad de los estadounidenses encuentra sus cimientos y referente en una historia que no debe ser olvidada. Los ciudadanos son los llamados a rehacer el país por el progreso de la nación que debe caminar hacia el bien común y hacia la necesidad de emprender con valentía.

Según explica Collins (21 de enero, 2009), «President Obama has made it clear that each of us has a role to perform, sacrifices to make in the great task of rebuilding the country».

\subsubsection{Afghanistan, Alliance, Defense, Enemy, Freedom, Friend, Iraq, Peace, Power, Security, Threat, War.}

Se trata en síntesis de una seguridad nacional basada en la responsabilidad de la actual generación de estadounidenses de liderar al mundo en la lucha contra las amenazas actuales. 
Esta lucha se expresa particularmente como deterrence, es decir, una disuasión con las armas de la diplomacia, cuya fuente se encuentra en el poder en su conjunto, valores, convicciones, pero también poderío militar. Pero aún más, a juicio del mandatario, se trata de un conjunto de elementos que están sujetos a los principios por los que lucharon los Padres Fundadores de la Nación estadounidense: el Estado de Derecho y los derechos humanos.

Básicamente el mandatario no olvida que se trata de la nación más poderosa del planeta y que aunque considera captar adeptos a su causa en función de la fuerza de sus convicciones y valores permanentes, solo serán amigos de Estados Unidos quienes estén dispuestos a asumir un pensamiento y actuar compatible. Especialmente, considerando que el país se encuentra en guerra en contra de una red de violencia y odio de largo alcance, dice expresamente que la nación no vacilará en defender su propio modo de vida. En resumen, el mandatario no habla desde el estrado de la ingenuidad.

Ahora bien, cuando el mandatario declara que Estados Unidos se sitúa en posición de liderazgo de amigos que luchan por la paz y la dignidad, indica una relación que no es igualitaria. Se trata de un liderazgo global autoasumido y proclamado, de un líder que no pide ayuda, sino que busca adeptos por la fuerza de sus convicciones. Destaca el hecho de que no menciona la Organización de las Naciones Unidas, ni la pa- labra consenso, aunque sí manifiesta el interés por trabajar con otras naciones. Asimismo, cabe destacar que el concepto de poder se relaciona también con el ámbito interno, en una crítica a la corrupción y al engaño que ha mermado las políticas y capacidades del país, obteniendo como resultado una crisis de confianza dentro de la Nación que ha incidido en el desarrollo de otras tres crisis que causan el cuestionamiento del correcto desarrollo de Estados Unidos.

Desde otra perspectiva, se observa que la conceptualización de una nueva era de la paz tiene alcances en el ámbito interno y externo. Al interior de Estados Unidos se intenta conciliar la convivencia de todas las culturas para alcanzar una unidad de propósito. Del discurso se desprende que es justamente por la heterogeneidad cultural que el país debe jugar un rol en la construcción de una nueva era de paz mundial en la medida en que el mundo se hace más pequeño y se revelan los aspectos comunes a toda la humanidad. De manera específica, el presidente se refiere a Irak y Afganistán indicando la relevancia de las guerras en su mandato, dejando en claro a la ciudadanía que no es un tema para evadir sino para enfrentar: entregando a los iraquíes el control de su propio país y construyendo la paz en Afganistán. En forma extensiva menciona expresamente al mundo musulmán, con el que se busca una nueva forma de avanzar en torno al interés y respeto mutuos. 


\section{Conclusiones}

El estudio indica que el discurso inaugural de la presidencia de Barack Obama es una convocatoria en que se busca integrar a un ente social compuesto por ciudadanos estadounidenses, que responden a los sujetos verbales Our, We, Us. Se podría decir que el líder electo en las urnas busca ampliar su base de convocatoria y asentarla en el pueblo apelando al sentimiento más que al pragmatismo, en el espíritu de unidad más que en la propuesta de soluciones concretas.

El mandatario hace un llamado apelando a la fe y determinación de los estadounidenses (sobre la que se sustenta la Nación) para superar las crisis actuales. Refuerza este llamado haciendo presente una de las enseñanzas de las generaciones precedentes: que la libertad y la paz se conquistan día a día. Sostiene que el panorama actual demanda de los americanos asumir que han entrado en una era de responsabilidad en la que será determinante el espíritu de servicio de los ciudadanos que crean que el mejor legado que pueden dejar a las futuras generaciones es la libertad y la paz.

La fórmula básica del líder es cohesionar al tejido social heterogéneo que integra la nación estadounidense reconociendo en el pueblo norteamericano la capacidad de superar las dificultades cuando enfrenta situaciones adversas.

En este marco general, identificamos que el discurso aborda la seguridad nacional en un sentido amplio. Esto se expresa en la única mención explícita del vocablo «security», lo que indica que a juicio del orador, la seguridad no consiste solamente en el poderío militar sino que incluye otros factores fundamentales tales como la justicia de la causa estadounidense, la fuerza del ejemplo y las cualidades atenuantes de la humildad y la moderación.

De la lectura del discurso se extrae una secuencia lógica que acentúa la aplicación de los valores y principios nacionales permanentes junto con la integración social y cultural de la nación haciendo hincapié en el desarrollo para enfrentar los problemas de seguridad, a saber:

1. Identificar la crisis de confianza como la base de las demás crisis que afectan a Estados Unidos.

2. Identificar como solución a la crisis de confianza el regreso a los principios y valores democráticos tradicionales del país. Asociado a ello se encuentra la lucha independentista de los padres de la patria, que lo sacrificaron todo por fundar un país en que imperen la ley y los derechos civiles. Fueron ellos quienes marcaron la senda de la paz y la libertad de los ciudadanos, quienes conquistaron el poder para entregarlo a la voluntad del pueblo. Por ende, ir en contra de los principios y valores democráticos es desconocer el motivo mismo de la nación.

Recuperando los principios democráticos del país se enfrentan problemas internos tales como la 
segregación y la relación gobiernopueblo. Desde otra perspectiva, se encaran los problemas de corrupción y las irregularidades del mercado. Asimismo, se considera que el gobierno debe estar al servicio del pueblo y que el mercado debe estar bajo control, particularmente cuando se esboza la idea de que la prosperidad es para todos y no solo para los prósperos.

3. El retorno a la democracia tiene como consecuencia el empleo responsable de los medios de poder del gobierno, la administración del poder, tanto al interior como al exterior, está sujeta a la rendición de cuentas 5 . Aplicado a la política internacional este principio implica que el poder militar queda acotado al imperio de la ley y los derechos civiles, ejercidos en forma responsable. Lo anterior repercute en forma positiva frente a la crisis de aliados en la medida en que deja de tener sentido aplicar el poder militar en forma autónoma, y que hay que hacerlo con el apoyo internacional convocando a los países del orbe a formar alianzas en función de valores y convicciones comunes, bajo el liderazgo de Estados Unidos.

4. Respecto de las crisis bélicas (Afganistán e Irak), manifiesta la necesidad de encontrar una solución en la construcción de la paz, más que en una victoria militar.

5 Referido a lo que comúnmente se entiende como accountability.
En suma, del discurso se deduce que la postura del mandatario ante las crisis actuales es que ellas se resuelven mediante la capacidad de los recursos humanos del país, considerando los principios y valores permanentes de la Nación y destacando que el puntal de desarrollo de Estados Unidos está en la fe y la determinación del pueblo, mosaico heterogéneo de culturas, que debe tener espíritu de servicio y asumir responsabilidades compartidas para superar los tiempos de crisis y entregar el legado de la libertad y paz a las futuras generaciones, como lo hicieron en su tiempo los Padres Fundadores del país.

Específicamente, respecto de la defensa puede sostenerse que sin criticar a la administración anterior, el presidente Obama solo menciona expresamente dos veces, primero para acotar su aplicación en función del imperio de la ley y los derechos humanos y luego para demostrar al concierto internacional que, como líder mundial, Estados Unidos no dará explicaciones sobre su modo de vida.

Obama señala en su discurso que tanto el gobierno como la ciudadanía deben velar por la defensa y la aplicación de los principios democráticos, tanto a nivel externo como interno porque de lo contrario las crisis se mantendrán o agravarán, con la posibilidad de que se hipoteque el futuro de las generaciones siguientes restringiendo la libertad y por ende la paz, por la que la nación estadounidense ha luchado desde el momento mismo de su fundación. 
Asimismo, pese a que dice en forma directa lo que persigue en Afganistán e Irak, no señala cómo va a lograr sus objetivos. Dejando en claro al respecto de que ahora más que nunca Estados Unidos y por ende los ciudadanos, deben velar primero por su desarrollo y seguridad más que preocuparse por temas externos. El retorno de la confianza debe entenderse como la solución que tendrá consecuencias transversales en los principales problemas que aquejan al país.

Es importante destacar que durante el discurso el mandatario no se refiere al gasto en defensa, que es uno de los puntos más criticados por la ciudadanía al gobierno. Omite la política que va a implementar respecto de este gran tema de Estado que la comunidad internacional ha visto y seguirá viendo como una gran problemática que se debe enfrentar.

Desde una perspectiva más amplia, observamos que el hilo conductor del mensaje es el concepto de la esperanza, el creer que no han menguado las capacidades de la ciudadanía de Estados Unidos y que la unión de propósito de este tejido social heterogéneo permitirá a este gran grupo de personas actuar responsablemente como nación asumiendo los desafíos actuales y buscando generar los cambios que permitan alcanzar una nueva era de paz.

Finalmente, y sin ánimo de formular juicios sobre el discurso del mandatario estadounidense, sus planteamientos son más conceptuales que concretos, constituyen una amplia invitación al pueblo a emprender una marcha unidos para enfrentar las dificultades del presente. Se trata de un discurso del líder a la masa, más orientado a un sentimiento de patria que a explicitar necesidades concretas de gestión, por lo tanto se mantiene la incertidumbre económica y el cambio de enfoque que propone en términos de seguridad carece de medidas concretas. Con todo, si bien se mantiene la incertidumbre política y económica, se establece la idea de que hay que enfrentarla en conjunto.

\section{Fuentes BibliográficAS}

«Bush Presidency Closes With 34\% Approval, 61\% Disapproval». Gallup, 14 de enero, 2009. En: http:/www.gallup.com/ poll/113770/Bush-Presidency-Closes-34Approval-61-Disapproval.aspx

Washington Post (20 de enero, 2009), «Inauguration Day»,Sección Editorial.

Berkowitz, Bruce (2008) Strategic Advantage: challengers, competitors, and threats to America's future, Washington D.C: Georgetown University Press.

Cohen, Roger (22 de enero, 2009) «The Age of Responsibility», The New York Times.

Collins, Gail (21 de enero, 2009), «Woodstock Without the Mud», The New York Times versión electrónica: http://www.nytimes.com/2009/01/22/opinion/22collins. html?th\&emc $=$ th

Friedman, George (19 de enero, 2009), «Obama Enters The Great Game». Strategic Forecasting.

Gellman, Barton (20 de enero, 2009), "Obama's Moment Arrives», Washington Post.

Jones, Jeffrey M. (26 de enero, 2009) «Obama’s Initial Approval Ratings in Historical Context», Gallup en: http:/www.gallup.com/ 
poll/113968/Obama-Initial-ApprovalRatings-Historical-Context.aspx

Krippendorff, Klaus, (1997) Metodología de análisis de contenido. Teoría y práctica, Barcelona, Ediciones Paidós Ibérica S.A.

Kristof, Nicholas D. (21 de enero, 2009), «The Remaking of America», The New York Times, versión electrónica: http://www.nytimes.com/2009/01/22/opinion/22kristof. html? th\&emc=th

Montero S., María Dolores (1998) La información periodística y su influencia social, Barcelona: Universidad Autónoma de Barcelona.

Obama, Barack. a (20 de enero, 2009). "Time has come to reaffirm our enduring spirit'». Transcripción del discurso inaugural pronunciado por el mandatario de Estados Unidos en la ceremonia de toma de posesión del cargo. Washington D.C.: Associated Press. Disponible el 20 de enero de 2009 en http://www.msnbc.msn.com/ id/28751183/

Pellegrini Ripamonti, Silvia, (1993) «Información, Comunicación y Defensa: marco conceptual de relaciones», Memorial del Ejército de Chile ( $\left.\mathrm{N}^{\circ} 442 / 1993\right)$, pp 22-41.

Priest, Dana (23 de enero, 2009). "Bush's 'War' On Terror Comes to a Sudden End", Washington Post.

Rodríguez Gómez, Gregorio; Javier Gil Flores y Eduardo García Jiménez. (1999) Metodología de la Investigación Cualitativa. Colección Biblioteca de Educación.: Ediciones Aljibe.
Ruiz Olabuénaga, José Ignacio (2003), Metodología de la investigación cualitativa, Bilbao: Universidad de Deusto ( $3^{\mathrm{a}}$ ed).

Tironi, Eugenio y Cavallo Ascanio. (2006) Comunicación Estratégica. Vivir en un mundo de señales, Santiago: Taurus Pensamiento, Aguilar Chilena de Ediciones S.A.

\section{Referencias bibliográficas}

«Palabras más repetidas en su discurso de toma de mando", Cuerpo Reportajes , $\mathrm{La}$ Tercera, 25 enero, 2009.

Obama, Barack. b (20 de enero, 2009). «Inaugural Address». Discurso inaugural pronunciado por el mandatario de Estados Unidos en la ceremonia de toma de posesión del cargo, Washington D.C. Difundido en vivo por CNN Chile.

Obama, Barack. c (20 de enero, 2009). «Discurso de toma de posesión del Presidente Barack Obama». Traducción al español del discurso inaugural pronunciado por el mandatario de Estados Unidos en la ceremonia de toma de posesión del cargo. Washington D.C. Publicado en la página web de la Embajada de Estados Unidos el 20 de enero de 2009 en: http://www.embajadaeeuu.cl/OpenNews/asp/pagDefault.as p argInstanciaId $=1 \& \arg$ NoticiaId $=4385$

Stolberg, Sheryl Gay (22 de enero, 2009), «On Day One, Obama Sets a New Tone», The New York Times. 